\title{
Observation of Chemistry in Protoplanetary Disks
}

\author{
Anne Dutrey \\ Observatoire de Bordeaux, 2 rue de l'observatoire, F-33270 Floirac, \\ France
}

\begin{abstract}
.
Protoplanetary disks are now intensively observed by $\mathrm{mm}$ arrays. While resolved images of $\mathrm{CO}$ rotation lines permit a better understanding of their physical structure, molecular surveys provided by current mm telescopes are currently sensitivity limited and do not allow a quantitative analysis of the chemical properties of disks. In this paper, I review the actual observational knowledge of the chemistry in the outer disks surrounding low and intermediate PMS stars and traced by mm data.
\end{abstract}

\section{Introduction}

Using the IRAM array, we are studying protoplanetary disks surrounding TTauri stars, and to a lesser extent Herbig Ae stars (Dutrey et al. 2003, Piétu et al. 2003) in a long term project. Recently, the multi-transition and multi-isotopes analysis of CO we have performed on the outer disk of DM Tau has allowed us to derive the vertical temperature gradient inside the disk (Dartois et al. 2003). The method is very promising because this guarantees some accuracy in the measurements of the physical parameters of disks, a necessary step before a detailed study of the disk chemistry. Current $\mathrm{mm}$ interferometers are sensitivity limited, they only permit us to access the physical conditions of outer disks and molecular survey are of limited quality. As a consequence, most of the chemical properties of disks are actually inferred from chemical models. On our side, the strategy is: i) to determine the geometry, ii) to derive the physical properties of disks from $\mathrm{CO}$ lines and dust maps, iii) then to use this knowledge to begin an observational approach of the disk chemistry. After a presentation of the $\chi^{2}$ minimization method and of main results obtained, I will discuss current observations of molecular lines in disks and the resulting status of the observable chemistry.

\section{Physical structure of disks inferred from CO line maps}

$\mathrm{CO}$ maps reveal that the disks are in Keplerian rotation and that many disks in Taurus-Auriga clouds are large with typical radii $R_{\text {out }} \simeq 300-800 \mathrm{AU}$.

Even if the current $\mathrm{mm}$ arrays are sensitivity limited, they can be used, in the case of the bright objects, to derive the main physical parameters of the disks with a reasonable accuracy. For this purpose, the transfer function of the interferometer, namely the "dirty beam" or the Fourier Transform of the $U V$ 
coverage must be properly taken into account in the analysis together with the thermal noise.

This can only be achieved by performing an analysis inside the $U V$ plane because an interferometer measures visibilities. In the image plane, the deconvolution which permits to remove the side-lobes of the "dirty beam" is a highly non-linear process. As a consequence, comparing disk models to observations of disks in the image plane is actually a method which looses significantly in accuracy. In most cases, this cannot allow us to define the more relevant model and only leads to limited results. This problem will be less important with ALMA since the sampling of the $U V$ plane will be significantly higher.

In Guilloteau \& Dutrey 1998, we present a method of minimization of disk parameters performed inside the $U V$ plane. We directly compare the observed visibilities to the model by calculating the distance $\left(\chi^{2}\right)$ between the observed visibilities and the Fourier transform of the model weighted by the thermal noise:

$$
\chi^{2}=\Sigma_{n} \Sigma_{i}\left(\operatorname{Re}\left(\bmod _{i, n}-\operatorname{Re}\left(o b s_{i, n}\right)\right)^{2} \times W_{i}+\Sigma_{n} \Sigma_{i}\left(\operatorname{Im}\left(\bmod _{i, n}-\operatorname{Im}\left(o b s_{i, n}\right)\right)^{2} \times W_{i}\right.\right.
$$

where $\operatorname{Re}(a)$ (resp. $\operatorname{Im}(a))$ is the real (resp. imaginary) part of the visibility $a$ and $a_{i, n}$ is the visibility $i$ for velocity channel $n$. The weight $W_{i}$ is derived from the system temperature $T_{s y s}$, the spectral resolution $\Delta \nu$, the integration time $\tau$, the effective collecting area of one antenna $A_{\text {eff }}$ and the loss of efficiency introduced by the correlator $\eta: W_{i}=\frac{1}{\sigma_{i}^{2}}$ with $\sigma_{i}=\frac{\sqrt{2} k T_{s y s}}{A_{\text {eff }} \eta \sqrt{\tau \Delta \nu}}$.

The disk model (Dutrey et al. 1994) is based on Pringle (1981): it is a geometrically thin disk in hydrostatic equilibrium with sharp inner and outer edges. Temperature, velocity and surface density assume power law radial dependencies. Moreover since the $\mathrm{J}=1-0$ and $\mathrm{J}=2-1$ transitions of $\mathrm{CO}$ are thermalized by collisions with $\mathrm{H}_{2}$, the radiative transfer is solved by integrating along the line-of-sight under assumption of LTE conditions. In LTE conditions, optically thick lines are direct tracers of the kinetic temperature at $\tau \simeq 1$ along the lineof-sight. The brightness temperature for optically thin lines is a function of the kinetic temperature and surface density. For the $\mathrm{J}=2-1$ lines, the brightness temperature is approximately proportional to $T_{K}(r) \Sigma(r)$. Due to the limited sensitivity of the data, the molecular abundance with respect to $\mathrm{H}_{2}$ is taken as a constant throughout the disk.

\subsection{Deriving physical parameters from a $\mathrm{mm}$ array}

In the analysis of the DM Tau disk, we found that for ${ }^{12} \mathrm{CO}, \tau \simeq 1$ is reached at typically 2-3 scale heights $H$ above the disk mid-plane (note that for "historical" reasons, in our definition, the scale height $H$ is $\sqrt{2}$ larger than in the definition of D'Alessio et al. 1999 or Chiang et al. 1997). We refer to this surface as the "CO surface". We have now applied the method to a sample of a dozen of TTauri disks and a few Herbig Ae objects (Dutrey et al. 2004, in prep.). In all cases, we find that the radial profile of the temperature, at the $\mathrm{CO}$ surface, is consistent with stellar heating in flared disks. We also find that the turbulence appears to be small, less than or equal to $0.1 \mathrm{~km} \cdot \mathrm{s}^{-1}$.

Since the ${ }^{13} \mathrm{CO} J=1-0$ and $\mathrm{J}=2-1$ have different opacities and sample different disk layers, a global analysis of these lines permit to derive the vertical 
kinetic temperature gradient. In the case of DM Tau (Dartois et al. 2003), the measured gradient is in agreement with disk models (e.g. D'Alessio et al. 1999). The mid-plane is cooler $(\sim 13 \mathrm{~K})$ than the CO disk surface $(\sim 30 \mathrm{~K}$ at $100 \mathrm{AU})$. This appears in the region of the disk where the dust is still optically thick to the stellar radiation while it is already optically thin to its own emission, around $r \sim 50-200$ AU in the DM Tau case. Beyond $r \geq 200$ AU where the dust becomes optically thin to both processes, the temperature profile appears isothermal vertically. More recently, we have applied this method to LkCa15 and MWC480, we also find evidence for a vertical temperature gradient of similar magnitude (Piétu et al. 2003).

A significant fraction of the DM Tau disk has a temperature which is below the $\mathrm{CO}$ freeze out point $(17 \mathrm{~K})$ but there remains enough $\mathrm{CO}$ in the gas phase to allow the $\mathrm{J}=2-1$ line of the main isotope to be optically thick. This is also the case for most of the sources of our sample.

\section{Towards Chemistry}

Using current $\mathrm{mm}$ arrays to detect lines of low surface brightness in disks is complicated by the existence of the continuum which is a significant fraction of the total flux density and difficult to subtract. Therefore, a large single-dish telescope appears to us better suited for line detections.

\subsection{Molecular surveys: unresolved observations}

In Dutrey et al. 1997, we used the IRAM 30-m telescope to make a molecular survey of the DM Tau and GG Tau disks. Today, in addition to ${ }^{13} \mathrm{CO}$ and $\mathrm{C}^{18} \mathrm{O}$, only the more abundant species after the carbon monoxyde are detected, like $\mathrm{HCO}^{+}, \mathrm{CN}, \mathrm{C}_{2} \mathrm{H}, \mathrm{CS}, \mathrm{HCN}, \mathrm{HNC}, \mathrm{H}_{2} \mathrm{CO}$, and more recently $\mathrm{DCO}^{+}$(HilyBlant et al. 2004, in prep.). By studying the excitation conditions of the various transitions observed in the DM Tau disk, we have estimated the $\mathrm{H}_{2}$ density and deduced molecular abundances indicating large depletion factors, ranging from 5 for $\mathrm{CO}$ to 100 for $\mathrm{H}_{2} \mathrm{CO}$ and $\mathrm{HCN}$, with respect to the abundances in the TMC1 cloud. It is important to mention that radicals such as $\mathrm{CN}$ or $\mathrm{C}_{2} \mathrm{H}$ are less depleted than most of the other species. Since we estimated the $\mathrm{H}_{2}$ density, we were also able to derive the total disk mass. The value we got is a factor 7 smaller than the total mass measured from the thermal dust emission at $1.3 \mathrm{~mm}$. These results, obtained from unresolved observations, assume a very simple isothermal disk model and suffer from several sources of uncertainties. A more detailed analysis taking into account the physical structure of the disk and the apparent sizes of molecular line emission estimated from resolved maps is needed to conclude on the possible origin of the mass discrepancy, e.g. uncorrect values for the gas-to-dust ratio, dust absorption coefficient, abundances...

We have recently extended the 30-m survey to more sources including disks of TTauri and Herbig Ae stars. We observe variations from one source to another. However, there is no clear difference in the chemical behavior of outer disks surrounding TTauri stars and Herbig Ae stars since we detect the same molecular species (Hily-Blant et al. 2004, in prep.). 


\section{Molecular chemistry with $\mathrm{mm}$ arrays}

\subsection{A photon-dominated chemistry}

The global analysis of the $\mathrm{CO}$ isotopomers in the DM Tau disk (Dartois et al. 2003) reveals that the ${ }^{13} \mathrm{CO}$ outer radius is significantly smaller than the outer radius deduced from ${ }^{12} \mathrm{CO}$ transitions:

- $R_{\text {out }}\left({ }^{12} \mathrm{CO}\right)=800 \pm 5 \mathrm{AU}$, obtained independently from the $\mathrm{J}=1-0$ and $\mathrm{J}=2-1$ data sets.

- $R_{\text {out }}\left({ }^{13} \mathrm{CO}\right)=640 \pm 15 \mathrm{AU}$, also obtained independently from the $\mathrm{J}=1-0$ and $\mathrm{J}=2-1$ data.

We also notice the same trends in LkCa15 and MWC480 (Piétu et al. 2004, in prep.).

In the case of DM Tau, by comparing the ratio of the surface density laws at both outer radii assuming $p \simeq 1.5$, we found that the difference in outer radii is compatible with selective photo-dissociation of CO (Dartois et al. 2003).

Taken together with the fact that radicals such as $\mathrm{CN}$ or $\mathrm{C}_{2} \mathrm{H}$ appear to be less under-abundant than other molecules, photon-dominated chemistry seems to play a significant role in the outer disks of TTauri stars, at least at disk surfaces and edges.

\subsection{Molecular mapping with $\mathrm{mm}$ arrays}

Interferometric molecular mapping of good quality are limited to the brighter molecular species such as $\mathrm{CO}, \mathrm{HCO}^{+}$or $\mathrm{CN}$ (Piétu et al. 2004, in prep).

There are however a few attempts to map strongly depleted species in disks such as the organic molecule $\mathrm{H}_{2} \mathrm{CO}$ in DM Tau (see Fig.1) or in LkCa15 (Aikawa et al. 2003). These data do not permit yet a detailed study. Moreover, the analysis is complicated by the fact that the line can be partially sub-thermal, requiring full radiative transfer code and several transitions to disentangle between excitation conditions and abundance gradient. This will be the domain of ALMA. We illustrate this point through Fig.1 where we present the channel maps of the $\mathrm{H}_{2} \mathrm{CO} 3_{13}-2_{12}$ line in DM Tau. The integration time with the IRAM array is about $\sim 12$ hours. The data are of limited quality and do not allow us a detailed analysis as in the case of the $\mathrm{CO}$ data. Obtaining data of same quality as $\mathrm{CO}$ for the $\mathrm{H}_{2} \mathrm{CO} 3_{13}-2_{12}$ line, will require with ALMA an integration time of $\sim 1$ hour on DM Tau. Hence, even with ALMA, the study of the chemistry in protoplanetary disks will be time consuming for less abundant species, e.g. deuterated molecules.

\section{Summary}

Due to limited sensitivity and to provide a better comparison with disk models, current $\mathrm{mm}$ interferometric observations cannot be analyzed without taking into account the transfer function of the instrument. Our analysis of a few TTauri and Herbig Ae disks directly performed inside the $U V$ plane allows us to conclude that: 


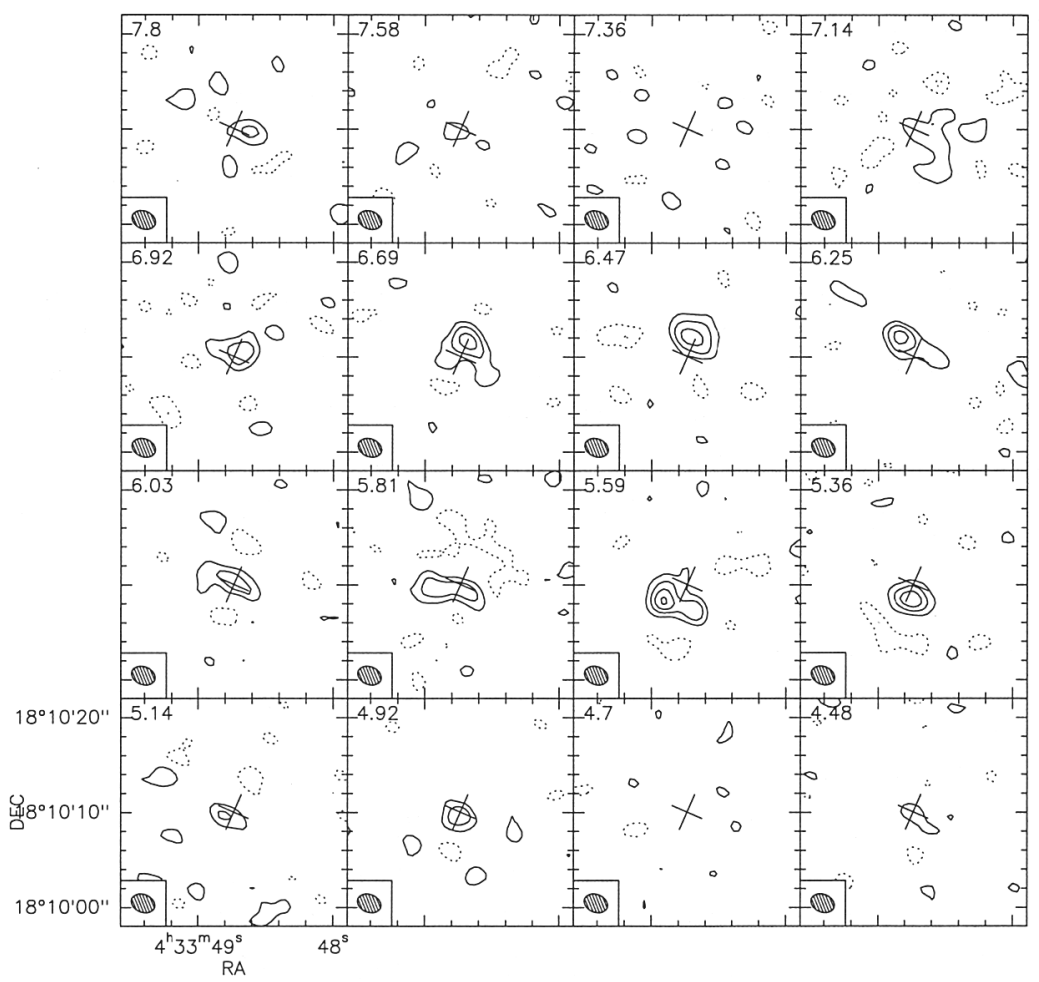

Figure 1. Channel maps of the $\mathrm{H}_{2} \mathrm{CO} 3_{13}-2_{12}$ transition observed with the IRAM Array in the DM Tau disk. The integration time is about $\sim 12$ hours. Beam size is $2.6^{\prime \prime} \times 1.8^{\prime \prime}, \mathrm{PA}=-110^{\circ}$, contours are $40 \mathrm{mJy} /$ Beam or $2 \sigma$. Note that the continuum of the source has not been removed. 
- The kinetic temperature at the CO disk surface is in agreement with the model of flared disks heated by the central star.

- Multi-line, multi-isotope analysis of CO reveal the existence of a vertical temperature gradient in outer disks which seems compatible with disk models, disk-mid planes being cooler than CO surfaces.

- CO line-widths are almost thermal and the turbulence appears negligible.

- The outer radius of disks in $\mathrm{CO}$ is governed by selective photo-dissociation. More generally, the presence of radicals such as $\mathrm{CN}$ or $\mathrm{C}_{2} \mathrm{H}$ suggests that photo-dissociation processes play a significant role, at least at the disk surface and edge.

Observational studies of the chemistry in disks are currently limited to detections of the more abundant species: $\mathrm{HCO}^{+}, \mathrm{CN}, \mathrm{C}_{2} \mathrm{H}, \mathrm{CS}, \mathrm{HCN}, \mathrm{HNC}$, $\mathrm{H}_{2} \mathrm{CO}$ and $\mathrm{DCO}^{+}$. Detailed molecular survey in outer and inner disks will be one key domain of ALMA but will require significant integration times.

Acknowledgments. I would like to thank Stéphane Guilloteau for a long term collaboration, Emmanuel Dartois, Vincent Piétu and Pierre Hily-Blant for many fruitful discussions.

\section{References}

Aikawa et al. 2003, PASP, 55, 11

D'Alessio, P. et al. 1999, ApJ, 527, 893

Chiang, E.I., \& Goldreich, P. 1997, ApJ, 490, 368

Dartois, E., Dutrey, A., Guilloteau, S., 2003, A\&A, 399, 773

Dutrey, A., Guilloteau, S., Simon M., 1994, A\&A, 286, 149

Dutrey, A., Guilloteau, S., Guélin M., 1997, A\&A, 317, L55

Dutrey, A., Guilloteau, S., Simon M., 2003, A\&A, 402, 1003

Dutrey, A., et al. 2004, in prep.

Hily-Blant et al. 2004, in prep.

Guilloteau, S., \& Dutrey A., 1998, A\&A, 339, 467

Piétu, V., Dutrey, A., Kahane, C., 2003, A\&A, 398, 565

Piétu, V., et al. 2004, in prep.

Pringle, J.E., 1981, ARA\&A, 19, 137 